\title{
Left atrial enlargement: A cause of stroke?
}

\author{
Larry B. Goldstein MD
}

See related research article by Bouzas-Mosquera and colleagues on page E657 and at www.cmaj.ca/lookup/doi/10.1503/cmaj.091688.

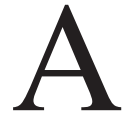
lthough there are a variety of established risk factors for ischemic stroke and its common pathophysiologies are well known, a specific cause remains undefined in $25 \%-40 \%$ of instances. That rate is influenced in part by the thoroughness of the poststroke evaluation. ${ }^{1}$ A cause, however, can remain undetermined in up to $12 \%$ of patients having an acute event, even with a full diagnostic assessment. ${ }^{2}$

At least part of the diagnostic uncertainty can be related to the impact of relatively unrecognized risk factors. For example, the most widely used stroke risk assessment tool, the Framingham stroke risk profile, includes the following wellrecognized stroke risk factors: age, systolic blood pressure, use of antihypertensive medications, diabetes mellitus, smoking, having cardiovascular disease, atrial fibrillation and left ventricular hypertrophy on electrocardiography. ${ }^{3}$ Although this tool is useful, many people have strokes despite a low Framingham stroke risk profile score, which does not incorporate a variety of less established risk factors. In this week's CMAJ, Bouzas-Mosquera and colleagues ${ }^{4}$ describe an independent, graded association between left atrial size on electrocardiography and stroke risk in women but not in men. Prior studies reported a similar relation between left atrial size and ischemic stroke risk in men, but not in women. ${ }^{5,6}$

The reason for the discrepancy among these studies is uncertain, and the findings need to be validated through replication in other patient populations. Bouzas-Mosquera and coauthors posit that the variability among reports might be a result of differences in study populations. Another possibility is that left atrial enlargement is a risk marker that only indirectly reflects one or a combination of risk factors. For example, left atrial enlargement is associated with an increased risk of atrial fibrillation, ${ }^{7}$ which is a major stroke risk factor that may become apparent only with prolonged and repeated cardiac monitoring.

Left atrial enlargement also might be a marker for other conditions that could increase stroke risk. For example, left atrial dysfunction can occur in patients with a patent foramen ovale. ${ }^{8}$ Patent foramen ovale (not assessed in the study by Bouzas-Mosquera and colleagues) is found more commonly in patients with cryptogenic stroke than in those with no history of stroke or in those who have stroke of determined cause. ${ }^{9}$ Paradoxical embolism through a patent foramen ovale may be important in the pathophysiology of acute stroke in patients with deep vein thrombosis, a relatively rare event.

The role of patent foramen ovale in patients without a source of embolism is less certain. There is no difference in the rate of recurrent stroke between groups of patients with otherwise cryptogenic stroke who do or do not have a patent foramen ovale, regardless of the type of medical treatment (antiplatelet medication v. anticoagulation). One randomized trial with results reported in abstract form (CLOSURE-1, 2011 International Stroke Conference) found no difference in the risk of recurrent stroke between patients treated with endovascular patent foramen ovale closure versus those receiving medical therapy. Additional clinical trials are in progress.

Studies also emphasize the important relation between lifestyle factors and stroke risk. Men and women who follow five lifestyle behaviours (not smoking, having a healthy diet, getting at least 30 min. per day of moderate or vigorous physical activity, having an optimal weight and consuming alcohol in moderation [no more than one alcoholic drink/day for women or one to two for men]) have an $80 \%$ lower risk of a first stroke as compared with those not following these behaviours, with increasing benefit depending on the number of lifestyle factors followed..$^{10}$ The study by BouzasMosquera and colleagues adjusted for cigarette smoking, but not these other lifestyle behaviours, which also affect the risk of cardiac disease.

\section{KEY POINTS}

- The cause of stroke is frequently uncertain.

- Traditional risk factors do not fully explain the cause of stroke in many people.

- Several studies report a novel association between left atrial enlargement and stroke, but vary regarding whether the relation is present in men as compared with women.

- Left atrial enlargement may be a risk marker rather than a risk factor.

All editorial matter in CMAJ represents the opinions of the authors and not necessarily those of the Canadian Medical Association. 
Several other novel risk factors not assessed in the study by Bouzas-Mosquera and colleagues are common to both stroke and cardiac disease. For example, a large number of studies point to a relation between both systemic inflammation and chronic infection and the risk of stroke as well as cardiac events. ${ }^{11}$ Trials of antibiotics, however, have not shown benefit in lowering the risk of recurrent coronary events. ${ }^{12,13}$ Similarly, treatments aimed at eliminating or controlling chronic infections have not proven to be helpful for the prevention of stroke. ${ }^{10}$ Inflammatory biomarkers such as high-sensitivity C-reactive protein and lipoproteinassociated phospholipase A2 are also independently associated with cardiovascular and stroke risk. One study found that treatment of patients with high blood levels of high-sensitivity Creactive protein with a statin led to a $44 \%$ reduction in the combined risk of cardiovascular events and stroke, with a $52 \%$ reduction in stroke risk, although the absolute rate of stroke was low. ${ }^{11}$

High levels of lipoprotein (a), a low-density lipoprotein particle, are associated with increased cardiovascular and stroke risk. Meta-analysis found that incident stroke was $22 \%$ higher in patients in the highest tertile of lipoprotein (a) compared with those in the lowest tertile. ${ }^{14}$ Levels of lipoprotein (a) can be lowered with niacin, but whether doing so reduces the risk of stroke has not been determined.

High homocysteine has also been associated with an increased risk of both cardiovascular events and stroke..$^{10}$ Randomized trials, however, have not found that lowering homocysteine with B vitamins reduces the risk of coronary events. Similarly, two trials found no reduction in recurrent stroke among patients with high homocysteine who were treated with B-vitamin supplementation. ${ }^{15,16}$ In contrast, analysis of data from another trial suggested that lowering of homocysteine was beneficial for prevention of secondary stroke. ${ }^{17}$ The role of B-vitamin supplementation to prevent a first or recurrent stroke remains uncertain. Current guidelines indicate that supplementation could be considered, although its benefit is not established..$^{10}$

There are numerous additional emerging potential risk factors for stroke..$^{10}$ Many, including several of those reviewed here, may also increase the risk of cardiac events and influence the development of left atrial enlargement. Whether left atrial enlargement represents a risk marker or a stroke risk factor, and whether interventions aimed specifically at reducing left atrial enlargement might be viable preventive interventions, requires further study.

\section{References}

1. Amarenco P, Bogousslavsky J, Caplan LR, et al. Classification of stroke subtypes. Cerebrovasc Dis 2009;27:493-501.

2. Michel P, Odier C, Rutgers M, et al. The Acute STroke Registry and Analysis of Lausanne (ASTRAL): design and baseline analysis of an ischemic stroke registry including acute multimodal imaging. Stroke 2010;41:2491-8.

3. D'Agostino RB, Wolf PA, Belanger AJ, et al. Stroke risk profile: adjustment for antihypertensive medication. The Framingham Study. Stroke 1994;25:40-3.

4. Bouzas-Mosquera A. Broullon FJ, Alvarez-Garcia N, et al. Left atrial size and risk for all-cause mortality and ischemic stroke. CMAJ 2011;183:1155.

5. Benjamin EJ, D'Agostino RB, Belanger AJ, et al. Left atrial size and the risk of stroke and death. The Framingham Heart Study. Circulation 1995;92:835-41.

6. Di Tullio MR, Sacco RL, Sciacca RR, et al. Left atrial size and the risk of ischemic stroke in an ethnically mixed population. Stroke 1999;30:2019-24.

7. Oliver JM, Gallego P, Gonzalez A, et al. Predisposing conditions for atrial fibrillation in atrial septal defect with and without operative closure. Am J Cardiol 2002;89:39-43.

8. Rigatelli G, Aggio S, Cardaioli P, et al. Left atrial dysfunction in patients with patent foramen ovale and atrial septal aneurysm: an alternative concurrent mechanism for arterial embolism? JACC Cardiovasc Interv 2009;2:655-62.

9. Overell JR, Bone I, Lees KR. Interatrial septal abnormalities and stroke - a meta-analysis of case-control studies. Neurology 2000;55:1172-9.

10. Goldstein LB, Bushnell CD, Adams RJ, et al. Guidelines for the primary prevention of stroke. A guideline for healthcare professionals from the American Heart Association/American Stroke Association. Stroke 2011;42:517-84

11. Ridker PM, Danielson E, Fonseca FAH, et al. Rosuvastatin to prevent vascular events in men and women with elevated Creactive protein. $N$ Engl J Med 2008;359:2195-207.

12. Grayston JT, Kronmal RA, Jackson LA, et. al. Azithromycin for the secondary prevention of coronary events. $N$ Engl J Med 2005;352:1637-45.

13. Cannon $\mathrm{CP}$, Braunwald $\mathrm{E}, \mathrm{McCabe} \mathrm{CH}$, et al. Antibiotic treatment of Chlamydia pneumoniae after acute coronary syndrome. N Engl J Med 2005;352:1646-54.

14. Smolders B, Lemmens R, Thijs V. Lipoprotein (a) and stroke: a meta-analysis of observational studies. Stroke 2007;38:1959-66.

15. Toole JF, Malinow MR, Chambless LE, et al. Lowering homocysteine in patients with ischemic stroke to prevent recurrent stroke, myocardial infarction, and death: the Vitamin Intervention for Stroke Prevention (VISP) randomized controlled trial. JAMA 2004;291:565-75.

16. The VITATOPS Trial Study Group. B vitamins in patients with recent transient ischaemic attack or stroke in the VITAmins TO Prevent Stroke (VITATOPS) trial: a randomised, double-blind, parallel, placebo-controlled trial. Lancet Neurol 2010;9:855-65.

17. Saposnik G, Ray JG, Sheridan P, et al. Homocysteine-lowering therapy and stroke risk, severity, and disability: additional findings from the HOPE 2 trial. Stroke 2009;40:1365-72.

Competing interests: Larry Goldstein has served on the Neurology Executive Committee and as site investigator for the RESPECT Trial (AGA), on the Steering Committee for the SPARCL Trial (Pfizer), on the Clinical Oversight Committee for the ACT-1 Trial (Abbott), as a consultant for Merck, Johnson and Johnson, Allergan and Boehringer-Ingelheim, and as a speaker for Bayer, Pfizer and MEDCON. His institution has received grants or has grants pending from the American Heart Association (ASA-Bugher Foundation Center for Stroke Prevention Research), the National Institutes of Health (CREST Trial; Stroke epidemiology and outcomes) and KB Reynolds (Stroke prevention in the Latino community). He has received payment from the Medical Tribune for manuscript preparation and royalties from Uptodate and Henry Stewart Talks.

Affiliations: Larry B. Goldstein is with the Duke Stroke Center, Duke University, and the Durham VA Medical Center, Durham, NC. 